\title{
Aptitud combinatoria y heterosis en híbridos de líneas endogámicas de maíz
}

\section{Combining ability and heterosis in hybrids from inbred lines corn}

\author{
Anselmo González-Torres ${ }^{1}$, J Guadalupe Luna-Ortega ${ }^{2 *}$, Miguel A Gallegos-Robles ${ }^{3}$, José L \\ García-Hernandez ${ }^{3}$, Pablo Preciado-Rangel ${ }^{1}$, César Guerrero-Guerrero ${ }^{4}$, Mario García-Carrillo . \\ ${ }^{1}$ Universidad Autónoma Agraria Antonio Narro, Unidad Laguna, Torreón, Coahuila, México. \\ ${ }^{2}$ Universidad Politécnica de la Región Laguna, carretera a Santa Teresa en el antiguo internado. \\ ${ }^{3}$ Facultad de Agricultura y Zootecnia, Universidad Juárez del Estado de Durango, Durango, México. \\ ${ }^{4}$ Centro de Estudios Tecnológicos Industriales y de Servicios No. 59. \\ ${ }^{*}$ Autor de correspondencia: lupe_lunao@yahoo.com.mx
}

Artículo científico recibido: 17 de octubre de 2015, aceptado: 11 de agosto de 2016

RESUMEN. El trabajo se realizó en dos etapas, en la primera se formaron las cruzas en el campo experimental de la Universidad Autónoma Agraria Antonio Narro Unidad Laguna (UAAAN-UL), y en la segunda la evaluación en el campo experimental de la UAAAN-UL en los ciclos agrícola primavera y verano, y en el ejido Niágara, en el municipio de Aguascalientes, Aguascalientes, en primavera. El material genético fueron 17 líneas endogámicas, cuatro de la UAAAN-UL, dos del INIFAP y 11 del CIMMYT. El objetivo fue estimar la aptitud combinatoria general (ACG) de las líneas, la aptitud combinatoria específica (ACE) y cuantificar los efectos genéticos y la heterosis de las cruzas. Para la ACG sobresalieron las líneas macho LAN-388P, LB-32 y LB-40, y las líneas hembras CML-319, CML-318 y CML-278; para la ACE los mayores valores lo tuvieron las cruzas LAN-123xCML-278 (3.28 t ha $\left.{ }^{-1}\right)$, LAN-123xCML-318 $(1.9 \mathrm{t}$ $\left.\mathrm{ha}^{-1}\right)$, LB-40xCML-319 (1.7 t ha $\left.{ }^{-1}\right)$ y LAN-388PxCML-264 $\left(1.52 \mathrm{t} \mathrm{ha}^{-1}\right)$. Mientras que el mayor rendimiento de grano, lo tuvieron las cruzas LB-40xCML-319 (14.49 $\mathrm{t} \mathrm{ha}^{-1}$ ), LB-32xCML-319 (14.17 t ha ${ }^{-1}$ ), LAN-388PxCML-264 (13.68 $\left.\mathrm{t} \mathrm{ha}^{-1}\right)$, LAN-123xCML-278 (13.55 $\mathrm{t} \mathrm{ha}^{-1}$ ) y LAN-388PxCML-278 $\left(13.44 \mathrm{t} \mathrm{ha}^{-1}\right)$. La varianza de dominancia superó a la varianza aditiva en rendimiento de grano $(R G)$ y en los principales componentes del rendimiento. Se encontraron efectos positivos de heterosis para rendimiento de grano con respecto al progenitor superior en las cruzas LB-40xCML-319 y LB-32 x CML-319.

Palabras clave: Zea mays L., probadores, efectos genéticos, híbridos, cruzas

ABSTRACT. The work was carried out in two stages. In the first, the crosses were formed in the Universidad Autónoma Agraria Antonio Narro Unidad Laguna experimental field (UAAAN-UL), and in the second the evaluation was carried out in the UAAAN-UL experimental field in the spring and summer agricultural cyles, and in the Niagara ejido, in the municipality of Aguascalientes, Aguascalientes, in the spring. The genetic material was 17 inbred lines, four from UAAAN-UL, two from INIFAP and 11 from CIMMYT. The objective was to estimate the general combining ability (GCA) and the specific combining ability (SCA) of the lines, and to quantify the genetic effects and heterosis of the crosses. For GCA, the male lines that stood out were LAN-388P, LB-32 and LB-40, and the female lines were CML-319, CML-318 and CML-278; for SCA, the highest values were obtained by the crosses LAN-123xCML-278 (3.28 $\left.\mathrm{t} \mathrm{ha}^{-1}\right)$, LAN-123xCML-318 (1.9 $\left.\mathrm{t} \mathrm{ha}^{-1}\right)$, LB-40xCML-319 $\left(1.7 \mathrm{t} \mathrm{ha}^{-1}\right)$ and LAN-388PxCML-264 (1.52 $\left.\mathrm{t} \mathrm{ha}^{-1}\right)$, while the best grain yield was obtained by the crosses LB-40xCML-319 (14.49 $\left.\mathrm{t} \mathrm{ha}^{-1}\right)$, LB-32xCML-319 (14.17 t ha-1), LAN-388PxCML-264 (13.68 $\mathrm{t} \mathrm{ha}^{-1}$ ), LAN-123xCML-278 (13.55 $\mathrm{t} \mathrm{ha}^{-1}$ ) and LAN-388PxCML-278 (13.44 $\mathrm{t} \mathrm{ha}^{-1}$ ). The dominance variance exceeded the additive variance in grain yield (GY) and in the main yield components. Positive effects of heterosis for grain yield were found with respect to the superior parent in the crosses LB-40xCML-319 and LB-32 x CML-319.

Key words: Zea mays L., testers, genetic effects, hybrids, crosses. 
González-Torres et al.

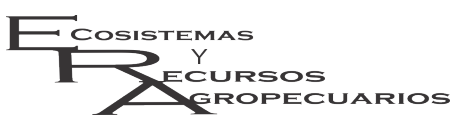

\section{INTRODUCCIÓN}

Las cruzas entre individuos de diferentes poblaciones o líneas con frecuencia tienen un comportamiento superior con respecto a sus padres, lo que se conoce como heterosis o vigor híbrido (Sylvain 2010). Los conceptos de aptitud combinatoria general (ACG) y aptitud combinatoria específica (ACE), se utilizan para expresar el comportamiento promedio de una línea en sus combinaciones híbridas, y para designar las combinaciones híbridas que resultan mejores o peores en relación con el promedio de la ACG de las líneas progenitoras (Sprague y Tatum 1942).

Cuando los efectos de aptitud combinatoria general son más importantes que los efectos de aptitud combinatoria específica, se recomienda mejorar a la población por selección recurrente; pero si los efectos de la aptitud combinatoria específica son los más importantes, la población debe mejorarse por hibridación (Reyes et al. 2004). Algunas líneas tienen la capacidad de combinar bien con otras líneas, lo que sugiere que tienen buena ACG, mientras que otras combinan bien sólo con otra línea en particular, lo que significa que tienen buena ACE. La ACG proporciona un enfoque para predecir efectos aditivos que contribuyen a la heterosis y la ACE juega un papel importante en la heterosis, la aptitud combinatoria se ha utilizado con éxito para identificar combinaciones superiores (Zhang et al. 2015).

El vigor híbrido se expresa mayormente en cruzamientos entre líneas de diferente origen genético; por ello es importante elegir el germoplasma adecuado para la producción de híbridos (Shawn 2012). De los primeros conceptos relacionados con la explotación de este fenómeno en maíz (Zea mays L.) destacan el de línea pura, que ha sido el punto de partida para el estudio de la heterosis y la aptitud combinatoria general y específica, la primera se asocia con el desempeño potencial de los progenitores y la segunda con el desempeño de las cruzas (De León et al. 2005). Por lo anterior el objetivo fue estimar los efectos de aptitud combinatoria general (ACG) de líneas progenitoras, la aptitud combinatoria específica (ACE), la heterosis de cruzas y el rendimiento.

\section{MATERIALES Y MÉTODOS}

El material genético utilizado fueron 17 líneas endogámicas de maíz, cuatro del programa de mejoramiento de la Universidad Autónoma Agraria Antonio Narro Unidad Laguna (UAAAN-UL), que se caracterizan por tener adaptación al trópico seco, con resistencia a plagas y temperaturas altas; dos líneas del Instituto Nacional de Investigaciones Forestales Agrícolas y Pecuarias (INIFAP), y 11 líneas del Centro Internacional de Mejoramiento de Maíz y Trigo (CIMMYT), adaptadas a clima tropical y subtropical húmedo, con resistencia a enfermedades y sequía. Las líneas macho fueron: LAN 447, que tiene 8 autofecundaciones, derivada de generaciones avanzadas del híbrido AN-447. La línea LAN 130, derivada de la cuarta generación de la cruza de H-507 x Celaya 2. La línea LAN 123, proveniente de una población criolla de la región de los Altos, en el municipio de Concepción, en Jalisco, con precocidad y tolerancia a sequía. La línea LAN 388P enana, con hojas anchas y verticales, generada de la tercera generación del híbrido AN-388. La línea LB-32 del INIFAP, derivada del híbrido H-353 y la línea LB40 , originada de la línea INIFAP-B-40. Mientras que las líneas hembra fueron: CML-319 CIMMyT, RecyW89(Cr.Arg/CIM.ShPINPH)6-3-2-4-B-B.

Línea CML-264 Pob21 CIMMYT, POB21C5F2193-1-B-8-1-3-BBB-F. Línea CML-254 Pob21 CIMMYT, TUXSEQ-149-2-BBB-1-BB-F. Línea CML313 CIMMYT, Pob501c0F6-3-3-2-1-B-B. Línea CML-273 Pob43 CIMMYT, (ACT643*43F7)-23-2-1-bb-F. Línea CML-247 Pool24 CIMMYT, (G24F119*G24F54)-6-4-1-1-BB-f. Línea CML271 Pob29 CIMMYT, pob29stec1hc25-6-4-BBB-F. Línea CML-278 Pob43 CIMMYT, DMANTES804353-1-1-b-1-BB-f. Línea CML-315 CIMMYT, Pob500P500c0F246-4-1-2-2-B*3. Línea CML-318 Recy W87[B810(66)S3/G24S2]-B-8-1-1-3-B*4 y línea CML-321 Pop 502 P502c0F1-1-3-1-B*4. Las cruzas se realizaron en el campo experimental de la UAAAN-UL, en Torreón, Coahuila; localizado entre 
los $24^{\circ} 30^{\prime}$ y $27^{\prime \prime}$ de LN, y los $102^{\circ}$ y $104^{\circ} 40^{\prime}$ de LO. Se realizaron las 66 cruzas posibles resultantes de los cruzamientos entre seis líneas macho y 11 líneas hembra, de acuerdo al diseño de apareamiento de Carolina del Norte II.

La evaluación de las cruzas se realizó en el campo experimental de la UAAAN-UL en los ciclos primavera y verano. Las siembras se realizaron en el mes de marzo y junio, mientras que en el ejido El Niágara, en el estado de Aguascalientes, la siembra fue en el mes de mayo. La siembra se realizó de forma manual, en todas las localidades se utilizó un diseño de bloques completos al azar con dos repeticiones. La parcela experimental fue de tres surcos de $2 \mathrm{~m}$ de largo con distancia entre surcos de 0.75 $\mathrm{m}$, con seis plantas por metro lineal, dando un total de 36 plantas por parcela, para tener una población aproximada de 85000 plantas ha $^{-1}$.

La fertilización se realizó con la fórmula 200 $\mathrm{N}, 90 \mathrm{P}, 00 \mathrm{~K}$, en dos etapas, el $50 \%$ de nitrógeno y todo el fósforo al momento de la siembra, y el resto del nitrógeno en la escarda realizada a los 42 $\mathrm{d}$ después de la siembra. Para el riego se usó cintilla, procurando que no faltara humedad; el control de malezas se realizó de forma manual, por medio de dos deshierbes antes de la escarda y tres después del aporcado del cultivo. Las plagas que se presentaron en las localidades fueron el gusano cogollero (Spodoptera frugiperda) y la pulga negra (Chaetocnema pulicaria); el primero se controló aplicando Decís (Deltametrina) en dosis de $1.0 \mathrm{~L} \mathrm{ha}^{-1}$ y para la pulga negra, se utilizó Lorsban (Organofosforado) a $1.0 \mathrm{~L} \mathrm{ha}^{-1}$.

La parcela útil fue el surco central, la cosecha se realizó a los 131 d después de la siembra, cuando el grano tenia aproximadamente $12.5 \%$ de humedad, omitiendo las plantas orilleras. Para luego pesar las mazorcas, medir la longitud de la mazorca (LM), diámetro de mazorca (DM), número de granos por hilera $(\mathrm{NGH})$ y número de hileras por mazorca (NHM). Las mazorcas se desgranaron y se pesó el grano en $\mathrm{kg}$ parcela ${ }^{-1}$ (RMZ), los datos de rendimiento se transformaron a tha ${ }^{-1}(R G)$ para su análisis. El análisis estadístico se realizó de acuerdo con el diseño de apareamiento de Carolina del Norte
II (Comstock y Robinson 1948) cuyo modelo lineal es: $\mathrm{Yijk}=\mu+\mathrm{Mi}+\mathrm{Hj}+\mathrm{MHij}+\mathrm{Rk}+£ i j k$.

Donde, $\mathrm{i}=1,2, \ldots \mathrm{m}$ (machos); $\mathrm{j}=$ $1,2, \ldots h$ (hembras); $k=1,2, \ldots . r$ (repeticiones); Yijk $=$ valor observado de la cruza entre el i-ésimo macho y la j-ésima hembra en la k-ésima repetición; $\mu=$ media general; $\mathrm{Mi}$ y $\mathrm{Hj}=$ efecto del i-ésimo macho y j-ésima hembra; $\mathrm{MHij}=$ efecto de la interacción del i-ésimo macho con la j-ésima hembra; £ijk = error experimental. La estimación de los efectos de aptitud combinatoria general (ACG) para los machos y hembras, y la aptitud combinatoria específica (ACE) para las cruzas, se realizó de acuerdo con Sprague y Tatum (1942).

La heterosis se estimó con respecto al promedio de los progenitores (h) y con respecto al progenitor superior ( $h$ '). De la siguiente forma: $h=$ $100(\mathrm{~F} 1-\mathrm{Pm}) / \mathrm{Pm}, \mathrm{h}^{\prime}=100$ (F1 - Ps)/Ps. Donde: $\mathrm{F} 1=$ es la primera generación de la cruza; $\mathrm{Pm}=\mathrm{el}$ progenitor medio $=(\mathrm{Pi}+\mathrm{Pj}) / 2 ; \mathrm{Ps}=$ el progenitor superior. Todos los análisis se realizaron con el paquete estadístico SAS V 6.11 (SAS 1988).

\section{RESULTADOS}

Los cuadrados medios del análisis de varianza, indican que la fuente de variación localidades ( $L$ ) mostró diferencias altamente significativas ( $p \leq$ 0.01 ) en todas las variables evaluadas (Tabla 1 ). Para machos (M), la mayoría de las variables tuvieron diferencias altamente significativas $(\mathrm{p} \leq$ 0.01), excepto para la longitud de mazorca (LMZ) que no presentó diferencias. En el caso de las hembras $(\mathrm{H})$ sólo las variables RG y NHM no tuvieron diferencias significativas. Para las cruzas $M^{*} H$, en las variables RG y RMZ no hubo diferencias significativas, mientras que para el DM y NHM se detectaron diferencias significativas $(\mathrm{p} \leq 0.05)$ y altamente significativas $(p \leq 0.01)$ en las variables LMZ y NGH. Para la interacción L*M se tuvieron diferencias altamente significativas $(p \leq 0.01)$ para el RG y significativas $(p \leq 0.05)$ para el RMZ. Para la interacción $L^{*} H$, no hubo diferencias significativas en ninguna de las variables evaluadas, mientras que en la interacción $L^{*} M * H$, sólo se tuvieron efec- 
tos significativos $(p \leq 0.05)$ en la variable NGH. Los coeficientes de variación encontrados estuvieron en un rango de $7.25 \%$ a $19.58 \%$.

Para el rendimiento de grano y mazorca de los progenitores macho, los mayores valores los tuvieron la línea LAN-388 P con 12.45 y $15.21 \mathrm{t} \mathrm{ha}^{-1}$, línea LB-32 con 11.66 y $13.65 \mathrm{t} \mathrm{ha}^{-1}$, y LB-40 con 11.46 y $13.69 \mathrm{t} \mathrm{ha}^{-1}$ (Tabla 2). Estos probadores también tuvieron efectos sobresalientes en las otras variables evaluadas. Para las líneas hembras del CIMMYT, los mayores valores promedio para RG y RMZ lo tuvieron la línea CML-319 con 11.41 y 15.37 t ha ${ }^{-1}$, línea CML-318 con 11.33 y $14.93 \mathrm{t} \mathrm{ha}^{-1}$, y la línea CML-278 con 10.96 y $14.27 \mathrm{t} \mathrm{ha}^{-1}$. Estos mismos progenitores hembra tuvieron los valores más altos para LMZ y DMZ (Tabla 2). El promedio de rendimiento de grano $(R G)$ y las variables evaluadas en las 15 mejores cruzas superiores se presenta en la Tabla 3, se observa que el rendimiento de grano osciló entre 12.18 y 14.49 t ha $^{-1}$, presentando los mayores valores las cruzas LB-40xCML-319 con $14.49 \mathrm{t} \mathrm{ha}^{-1}$, cruza LB-32xCML-319 con $14.17 \mathrm{t}$ $\mathrm{ha}^{-1}$, cruza LAN-388PxCML-264 con $13.68 \mathrm{t} \mathrm{ha}^{-1}$, cruza LAN-123xCML-278 con $13.55 \mathrm{t} \mathrm{ha}^{-1}$ y cruza LAN-388PxCML-278 con $13.44 \mathrm{t} \mathrm{ha}^{-1}$. Los efectos de aptitud combinatoria general (ACG) para el rendimiento de grano de las líneas macho, se muestran en la Tabla 4. De las líneas de la UAAANUL, sólo la línea LAN-388P presentó valores altos de ACG con $2.37 \mathrm{t} \mathrm{ha}^{-1}$, en tanto que en las líneas del INIFAP sobresalen la LB-32 y LB40 que tuvieron $A C G$ de 1.58 y $1.38 \mathrm{t} \mathrm{ha}^{-1}$. Para las líneas del CIMMYT usadas como hembras, las líneas con los mayores valores de ACG fueron la CML-319 con $1.33 \mathrm{t} \mathrm{ha}^{-1}$, CML-318 con $1.25 \mathrm{t}$ $\mathrm{ha}^{-1}$ y CML-278 con $0.88 \mathrm{t} \mathrm{ha}^{-1}$ (Tabla 4). Los valores más altos de ACE (Tabla 5) para rendimiento de grano y variables evaluadas en las 15 cruzas de mayores valores lo tuvieron la cruza LAN-123xCML278 con $3.28 \mathrm{t} \mathrm{ha}^{-1}$, LAN-123xCML-318 con 1.9 $\mathrm{t} \mathrm{ha}^{-1}$, LB-40xCML-319 con $1.7 \mathrm{t} \mathrm{ha}^{-1}$ y LAN$388 \mathrm{P} \times \mathrm{CML}-264$ con $1.52 \mathrm{t} \mathrm{ha}^{-1}$. Mientras que las cruzas LAN-388PxCML318, LAN-388PxCML-319 y LB-40xCML-318 tuvieron efectos negativos de ACE para RG.
La heterosis con relación al progenitor medio (h) y progenitor superior ( $h$ ') para cada una de las variables evaluadas, se presenta en la Tabla 6 , donde se observa que el valor máximo y mínimo de heterosis, para rendimiento de grano (RG) para al progenitor medio osciló entre 11 y $27 \%$. Mientras que la heterosis con respecto al mejor progenitor tuvo valores de 2.0 a $26 \%$.

\section{DISCUSIÓN}

La significancia encontrada en el análisis de varianza combinado para localidades, señala el efecto diferencial de los factores ambientales sobre el comportamiento de las cruzas evaluadas, lo que contrasta la respuesta genotípica de las cruzas en las localidades (Manjarrez et al. 2014). Para las líneas machos y hembras, la significancia encontrada indica la diversidad alélica en las mismas, lo cual es una condición necesaria para obtener híbridos sobresalientes (Wong et al. 2007, Guillen-De la Cruz et al. 2009). Estas diferencias denotan la diversidad genética del material evaluado, lo que coincide con lo señalado por Antuna et al. (2003) y De la Cruz et al. (2003). La significancia en la interacción de localidades por machos, indica que los machos en sus combinaciones hibridas interactúan de forma diferente con el ambiente de las localidades, lo que no se observó en la interacción de localidades por hembras, e indica que las hembras en sus combinaciones hibridas interactúan de manera similar con el ambiente de esas localidades. La consideración del componente ambiental es un aspecto importante que no puede pasarse por alto, ya que influye en el progreso y avance en la selección de materiales sobresalientes (Bello y Olaoye 2009). Para la interacción $\mathrm{L}^{*} \mathrm{M} * \mathrm{H}$ solo la variable $\mathrm{NGH}$ mostró significancia, señalando que en esta variable las cruzas interactúan de manera diferente con el ambiente (Gutiérrez et al. 2002). La variabilidad genética entre poblaciones es un factor importante para la obtención de líneas sobresalientes partir de las cuales se pueden obtener híbridos de maíz de alto rendimiento (Wong et al. 2007).

Las líneas probadoras LAN-388P, LB-32 y LB- 
González-Torres et al.

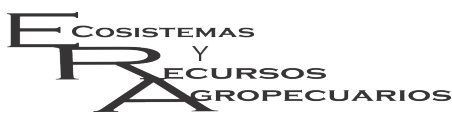

Aptitud combinatoria y heterosis en maiz

Ecosist. Recur. Agropec.

4(11):223-232,2017

Tabla 1. Cuadrados medios del análisis de varianza de un diseño Carolina del Norte II.

\begin{tabular}{llllllll}
\hline $\mathrm{FV}$ & $\mathrm{GL}$ & $\mathrm{RG} \mathrm{t} \mathrm{ha}^{-1}$ & $\mathrm{RMZ} \mathrm{t} \mathrm{ha}^{-1}$ & $\mathrm{LMZ} \mathrm{Cm}$ & $\mathrm{DMZ} \mathrm{Cm}$ & $\mathrm{NGH}$ & $\mathrm{NHM}$ \\
\hline $\mathrm{L}$ & 2 & $76.20^{* *}$ & $113.03^{* *}$ & $190.51^{* *}$ & $1.98^{* *}$ & $206.30^{* *}$ & $34.23 * *$ \\
$\mathrm{R} / \mathrm{L}$ & 3 & $271.58^{* *}$ & $60.74^{* *}$ & 4.27 & $1.17^{* *}$ & $242.32^{* *}$ & 2.33 \\
$\mathrm{M}$ & 5 & $275.13^{* *}$ & $39.80^{* *}$ & 26.66 & $0.90^{* *}$ & $115.39^{* *}$ & $38.10^{* *}$ \\
$\mathrm{H}$ & 10 & 24.48 & $30.14^{* *}$ & $92.81^{* *}$ & $0.39^{* *}$ & $131.89^{* *}$ & 7.08 \\
$\mathrm{M}^{*} \mathrm{H}$ & 50 & 13.12 & 11.00 & $70.86^{* *}$ & $0.19^{*}$ & $30.42^{* *}$ & $7.20^{*}$ \\
$\mathrm{~L}^{*} \mathrm{M}$ & 10 & $70.60^{* *}$ & $18.51^{*}$ & 27.53 & 0.17 & 23.91 & 3.25 \\
$\mathrm{~L}^{*} \mathrm{H}$ & 20 & 7.16 & 9.98 & 10.89 & 0.04 & 21.95 & 3.11 \\
$\mathrm{~L}^{*} \mathrm{M}^{*} \mathrm{H}$ & 100 & 5.44 & 7.71 & 14.16 & 0.09 & $23.35^{*}$ & 2.73 \\
Error & 194 & 14.44 & 9.63 & 30.89 & 0.11 & 17.05 & 4.57 \\
Media & & 10.08 & 13.7 & 18.78 & 4.7 & 38.21 & 14.57 \\
$\mathrm{CV}$ & & 17.67 & 12.64 & 19.58 & 7.25 & 10.80 & 14.68 \\
\hline ** = Significativo &
\end{tabular}
de libertad, $C V=$ Coeficiente de variación, $R G=$ Rendimiento de grano, $R M Z=$ Rendimiento de mazorca, $\mathrm{LMZ}=$ Longitud de la mazorca, $\mathrm{DMZ}=$ Diámetro de la mazorca, $\mathrm{NGH}=$ Número de granos por hilera, NHM= Número de hileras por mazorca.

Tabla 2. Comportamiento promedio de líneas endogámicas de maíz usadas como macho y hembra.

\begin{tabular}{|c|c|c|c|c|c|c|}
\hline Línea & $\mathrm{RG}_{\mathrm{t}} \mathrm{ha}^{-1}$ & $\mathrm{RMZ} \mathrm{t} \mathrm{ha}^{-1}$ & $\mathrm{LMZ} \mathrm{Cm}$ & DMZ cm & $\mathrm{NGH}$ & NHM \\
\hline \multicolumn{7}{|c|}{ Machos } \\
\hline LAN 447 & $7.81 \mathrm{c}$ & $13.47 \mathrm{~b}$ & $19.52 a$ & $4.65 b c$ & $37.59 c$ & $14.12 b c$ \\
\hline LAN 130 & $7.78 \mathrm{c}$ & $12.95 b$ & $19.33 a$ & $4.72 \mathrm{~b}$ & $38.05 \mathrm{~b}$ & $13.72 \mathrm{c}$ \\
\hline LAN 123 & $9.39 \mathrm{~b}$ & $13.30 \mathrm{~b}$ & $19.11 a$ & $4.64 b c$ & $39.23 a b$ & $14.48 \mathrm{~b}$ \\
\hline LAN 388P & $12.45 \mathrm{a}$ & $15.21 \mathrm{a}$ & $18.62 a$ & $4.92 \mathrm{a}$ & $38.95 \mathrm{ab}$ & $15.95^{a}$ \\
\hline LB 32 & $11.66 \mathrm{a}$ & $13.65 \mathrm{~b}$ & $18.25 a$ & $4.72 b$ & $35.95 \mathrm{~d}$ & $14.76 \mathrm{~b}$ \\
\hline LB 40 & $11.46 \mathrm{a}$ & $13.69 \mathrm{~b}$ & 17.91a & $4.58 c$ & $39.50^{a}$ & $14.43 \mathrm{bc}$ \\
\hline CML-319 & $11.41 \mathrm{a}$ & $15.37 a$ & $20.72 a b$ & 4.64def & $37.08 \mathrm{cde}$ & $14.26 \mathrm{bc}$ \\
\hline CML-264 & $9.79 a b c$ & $13.77 \mathrm{bcd}$ & $19.25 b c$ & $4.70 \mathrm{bcdef}$ & $41.31 a$ & $14.75 a b c$ \\
\hline CML-254 & $8.81 c$ & $12.39 \mathrm{~d}$ & $17.66 \mathrm{c}$ & $4.55 f$ & $37.61 \mathrm{bdc}$ & $14.52 \mathrm{abc}$ \\
\hline CML-313 & $9.66 \mathrm{abc}$ & $13.04 \mathrm{~cd}$ & $18.45 b c$ & 4.62def & $35.72 \mathrm{de}$ & $14.19 \mathrm{bc}$ \\
\hline CML-273 & $10.09 a b c$ & $14.23 \mathrm{abc}$ & $19.14 b c$ & $4.80 \mathrm{abc}$ & $38.29 b c$ & 14.99ab \\
\hline CML-247 & $10.04 \mathrm{abc}$ & $13.34 \mathrm{~cd}$ & $17.43 c$ & 4.77abcd & $37.89 \mathrm{bc}$ & $14.37 \mathrm{bc}$ \\
\hline CML-271 & $9.89 \mathrm{abc}$ & $13.17 \mathrm{~cd}$ & $16.96 c$ & $4.59 \mathrm{ef}$ & $35.19 \mathrm{e}$ & 14.99ab \\
\hline CML-278 & $10.96 \mathrm{ab}$ & $14.27 \mathrm{abc}$ & $17.37 \mathrm{c}$ & $4.84 a b$ & $41.22 \mathrm{a}$ & $14.25 b c$ \\
\hline CML-315 & $9.73 a b c$ & $13.50 \mathrm{bcd}$ & $22.28 a$ & 4.72abcde & $39.11 \mathrm{~b}$ & 14.54abc \\
\hline CML-318 & $11.33 a$ & 14.93ab & $19.41 b c$ & $4.87 a$ & $38.31 \mathrm{bc}$ & $15.48^{a}$ \\
\hline CML-321 & $9.25 b c$ & $12.78 \mathrm{~d}$ & $18.03 c$ & $4.65 \mathrm{cdef}$ & $38.58 \mathrm{bc}$ & $13.96 c$ \\
\hline
\end{tabular}

Medias con letras iguales no son estadísticamente diferentes (DMS, 0.05), RG = Rendimiento de grano, $\mathrm{RMZ}=$ Rendimiento de mazorca, $\mathrm{LMZ}=$ Longitud de la mazorca, $\mathrm{DMZ}=$ Diámetro de la mazorca, $\mathrm{NGH}=$ Número de granos por hilera, $\mathrm{NHM}=$ Número de hileras por mazorca.

40 fueron los más sobresalientes para el rendimiento de grano. Dentro de las hembras, sobresalen las líneas CML-319, CML-318 y CML-278 para RG, RMZ, LMZ y DMZ, mientras que los componentes que contribuyeron en menor medida al rendimiento de grano para las hembras fue el número de granos por hilera y el número de hileras por mazorca; sin embargo, la línea hembra CML-315 mostró el valor más alto para $\mathrm{LMZ}$, mientras que la línea CML-264 tuvo el valor más alto para NGH. Al combinar líneas con alto rendimiento de grano y valores de LMZ, DMZ, NGH y NHM el resultado podría ser un mayor número de granos por planta y por unidad de superficie. Al respecto Echarte et al. (2004) relacionaron estos componentes con un mayor rendimiento. Sin embargo, algunas líneas machos y hembras mostraron superioridad para rendimiento de grano y no para rendimiento de mazorca, al respecto, Rehman et al. (2011) señalan que correlaciones tanto positivas como negativas y significativas se han observado entre rendimiento de mazorca y rendimiento de grano, ya que existen otras características que provocan desviaciones, siendo la longitud de mazorca (LMZ) y el diámetro de la mazorca (DMZ), factores que permite un acomodo mayor de hileras y de granos 
González-Torres et al.

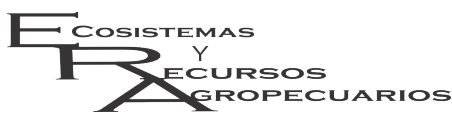

Aptitud combinatoria y heterosis en maiz

Ecosist. Recur. Agropec.

4(11):223-232,2017

Tabla 3. Promedios de las 15 mejores cruzas de maíz para seis variables agronómicas evaluadas.

\begin{tabular}{lllllll}
\hline Cruzas & $\mathrm{RG} \mathrm{tha}^{-1}$ & $\mathrm{RMZ} \mathrm{t} \mathrm{ha}^{-1}$ & $\mathrm{LMZ} \mathrm{Cm}$ & $\mathrm{DMZcm}$ & $\mathrm{NGH}$ & $\mathrm{NHM}$ \\
\hline LB-40XCML-319 & 14.49 & 17.06 & 20.98 & 4.63 & 37.00 & 13.92 \\
LB-32XCML-319 & 14.17 & 16.56 & 21.78 & 4.67 & 37.17 & 15.10 \\
LAN 388PXCML-264 & 13.68 & 16.23 & 18.87 & 4.92 & 42.00 & 13.97 \\
LAN 123XCML-278 & 13.55 & 16.15 & 17.67 & 4.98 & 40.67 & 14.75 \\
LAN 388PXCML-278 & 13.44 & 16.00 & 17.77 & 5.00 & 42.33 & 15.73 \\
LAN 388PXCML-247 & 13.39 & 16.13 & 19.53 & 5.05 & 38.67 & 15.03 \\
LAN 388PXCML-318 & 13.35 & 15.53 & 18.48 & 5.07 & 37.00 & 17.35 \\
LAN 388PXCML-319 & 13.29 & 15.74 & 19.52 & 4.85 & 37.00 & 16.87 \\
LAN 388PXCML-271 & 12.77 & 14.96 & 17.72 & 4.97 & 37.83 & 15.73 \\
LB-32XCML-271 & 12.63 & 14.83 & 18.85 & 4.73 & 31.83 & 15.93 \\
LB-40XCML-318 & 12.61 & 15.19 & 19.98 & 4.77 & 40.67 & 14.40 \\
LAN 123XCML-318 & 12.54 & 14.86 & 18.52 & 4.82 & 42.17 & 15.73 \\
LAN 388PXCML-313 & 12.41 & 14.36 & 22.27 & 4.88 & 37.00 & 15.73 \\
LB-32XCML-273 & 12.23 & 13.73 & 17.60 & 4.75 & 35.33 & 14.75 \\
LAN 388PXCML-321 & 12.18 & 14.41 & 17.92 & 4.90 & 39.17 & 15.38 \\
\hline DMS (p $\leq 0.05)$, RG= Rendimiento de grano, RMZ= Rendimiento de mazorca, LMZ= Lon- \\
gitud de la mazorca, DMZ= Diámetro de la mazorca, NGH= Número de granos por hilera, \\
NHM= Número de hileras por mazorca.
\end{tabular}

Tabla 4. Aptitud Combinatoria General (ACG) de líneas macho y hembras.

\begin{tabular}{lrcrrrr}
\hline Líneas & $\mathrm{RG} \mathrm{t} \mathrm{ha}^{-1}$ & $\mathrm{RMZ} \mathrm{t} \mathrm{ha}^{-1}$ & $\mathrm{LMZ}$ Cm & $\mathrm{DMZ} \mathrm{cm}$ & $\mathrm{NGH}$ & $\mathrm{NHM}$ \\
\hline \multicolumn{7}{c}{ Machos } \\
LAN 447 & -2.27 & -0.23 & 0.74 & -0.05 & -0.62 & -0.45 \\
LAN 130 & -2.3 & -0.75 & 0.55 & 0.02 & -0.16 & -0.85 \\
LAN 123 & -0.69 & -0.4 & 0.33 & -0.06 & 1.02 & -0.09 \\
LAN 388P & 2.37 & 1.51 & -0.16 & 0.22 & 0.74 & 1.38 \\
LB-32 & 1.58 & -0.05 & -0.53 & 0.02 & -2.26 & 0.19 \\
LB-40 & 1.38 & -0.01 & -0.87 & -0.12 & 1.29 & -0.14 \\
& & Hembras & & & & \\
CML-319 & 1.33 & 1.67 & 1.94 & -0.06 & -1.13 & -0.31 \\
CML-264 & -0.29 & 0.07 & 0.47 & 0.00 & 3.20 & 0.18 \\
CML-254 & -1.27 & $-1-31$ & -1.12 & -0.15 & -0.60 & -0.05 \\
CML-313 & -0.42 & -0.66 & -0.33 & -0.08 & -2.49 & -0.38 \\
CML.273 & 0.01 & 0.53 & 0.36 & 0.10 & 0.08 & 0.42 \\
CML-247 & -0.04 & -0.36 & -1.35 & 0.07 & -0.32 & -0.20 \\
CML-271 & -0.19 & -0.53 & -1.82 & -0.11 & -3.02 & 0.42 \\
CML-278 & 0.88 & 0.57 & -1.41 & 0.14 & 3.01 & -0.32 \\
CML-315 & -0.35 & -0.20 & 3.50 & 0.02 & 0.90 & -0.03 \\
CML-318 & 1.25 & 1.23 & 0.63 & 0.17 & 0.10 & 0.91 \\
CML-321 & -0.83 & -0.92 & -0.75 & -0.05 & 0.37 & -0.61 \\
\hline RG Rendin
\end{tabular}

$\mathrm{RG}=$ Rendimiento de grano, $\mathrm{RMZ}=$ Rendimiento de mazorca, $\mathrm{LMZ}=$ Longitud de la mazorca, $\mathrm{DMZ}=$ Diámetro de la mazorca, $\mathrm{NGH}=$ Número de granos por hilera, $\mathrm{NHM}=$ Número de hileras por mazorca.

por hileras, lo que impacta en un mayor número de granos en la mazorca (Wong et al. 2007). El peso de grano y las características asociadas con mayor número de granos, son las que definen el rendimiento, ya que existe la posibilidad de que al conjuntar por recombinación y selección algunas líneas contrastantes, se puedan tener patrones heteróticos sobresalientes, pues algunos híbridos altamente rendidores, lo fueron no sólo por la heterosis, sino también por factores hereditarios como la interacción múltiple de genes de tipo aditivo, lo que señala la conveniencia de conocer la importancia de cada componente genético (Wong et al. 2007).

Los machos LAN-388P, LB-32, LB-40 y las hembras CML-319, CML-318 y CML-278 fueron las líneas que más participación tuvieron en las cruzas sobresalientes, por lo que al conjugar genes favorables para la mayoría de las variables evaluadas se tuvieron cruzas sobresalientes, como lo señala De la Cruz et al. (2010). En las 15 mejores 
González-Torres et al.

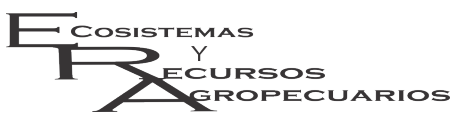

Aptitud combinatoria y heterosis en maiz

Ecosist. Recur. Agropec.

4(11):223-232,2017

Tabla 5. Aptitud combinatoria específica (ACE) de las 15 mejores cruzas de maíz, para seis características agronómicas evaluadas.

\begin{tabular}{lrrrrrr}
\hline Cruzas & $\mathrm{RG} \mathrm{t} \mathrm{ha}^{-1}$ & $\mathrm{RMZ} \mathrm{t} \mathrm{ha}^{-1}$ & $\mathrm{LMZ} \mathrm{cm}$ & $\mathrm{DMZ} \mathrm{cm}$ & $\mathrm{NGH}$ & $\mathrm{NHM}$ \\
\hline LB-40XCML-319 & 1.70 & 1.70 & 1.13 & 0.11 & -1.37 & -0.20 \\
LB-32XCML-319 & 1.18 & 1.24 & 1.59 & 0.01 & 2.35 & 0.65 \\
LAN 388PXCML-264 & 1.52 & 0.95 & -0.22 & 0.00 & -0.05 & -2.16 \\
LAN 123XCML-278 & 3.28 & 2.28 & -0.03 & 0.20 & -1.57 & 0.59 \\
LAN 388PXCML-278 & 0.11 & 0.22 & 0.56 & -0.06 & 0.37 & 0.10 \\
LAN 388PXCML-247 & 0.98 & 1.28 & 2.26 & 0.06 & 0.04 & -0.72 \\
LAN 388PXCML-318 & -0.35 & -0.91 & -0.77 & -0.02 & -2.05 & 0.49 \\
LAN 388PXCML-319 & -0.49 & -1.14 & -1.04 & -0.01 & -0.82 & 1.23 \\
LAN 388PXCML-271 & 0.51 & 0.28 & 0.92 & 0.16 & 1.90 & -0.64 \\
LB-32XCML-271 & 1.16 & 1.71 & 2.42 & 0.12 & -1.10 & 0.75 \\
LB-40XCML-318 & -0.10 & 0.27 & 1.44 & 0.02 & 1.07 & -0.94 \\
LAN 123XCML-318 & 1.90 & 0.33 & -1.22 & 0.01 & 2.84 & 0.34 \\
LAN 388PXCML-313 & 0.38 & -0.19 & 3.98 & 0.04 & 0.54 & 0.16 \\
LB-32XCML-273 & 0.56 & -0.45 & -1.01 & -0.07 & -0.70 & -0.43 \\
LAN 388PXCML-321 & 0.56 & 0.12 & 0.05 & 0.03 & -0.15 & 0.04 \\
\hline RG= Rendimiento de grano, RMZ= Rendimiento de mazorca, LMZ= Longitud de la mazorca, \\
DMZ= Diámetro de la mazorca, NGH= Número de granos por hilera, NHM= Número de hileras \\
por mazorca.
\end{tabular}

Tabla 6. Heterosis en base al progenitor medio (h) y al mejor progenitor ( $h$ '), de las variables evaluadas en las cruzas directas.

\begin{tabular}{|c|c|c|c|c|c|c|c|c|c|c|c|c|}
\hline \multirow[t]{2}{*}{ Cruza } & \multicolumn{2}{|c|}{$\mathrm{RG} \mathrm{t} \mathrm{ha}^{-1}$} & \multicolumn{2}{|c|}{$\mathrm{RMZ} \mathrm{t} \mathrm{ha}^{-1}$} & \multicolumn{2}{|c|}{$\mathrm{LMZ} \mathrm{Cm}$} & \multicolumn{2}{|c|}{ IDMZ cm } & \multicolumn{2}{|c|}{$\mathrm{NGH}$} & \multicolumn{2}{|c|}{ NHM } \\
\hline & $\mathrm{h}$ & $\mathbf{h}^{\prime}$ & $\mathrm{h}$ & $\mathbf{h}^{\prime}$ & $\mathrm{h}$ & $\mathbf{h}^{\prime}$ & $\mathrm{h}$ & $\mathbf{h}^{\prime}$ & $\mathrm{h}$ & $\mathbf{h}^{\prime}$ & $\mathrm{h}$ & $\mathrm{h}^{\prime}$ \\
\hline LB-40XCML-319 & 27 & 26 & 17 & 11 & 9 & 1 & 1 & 0 & -3 & -6 & -3 & -4 \\
\hline LB-32XCML-319 & 23 & 22 & 14 & 8 & 12 & 5 & 0 & -1 & 2 & 0 & 4 & 2 \\
\hline LAN-388PXCML-264 & 23 & 10 & 12 & 7 & 0 & -2 & 2 & 0 & 5 & 2 & -9 & -12 \\
\hline LAN-123XCML-278 & 33 & 24 & 17 & 13 & -3 & -8 & 5 & 3 & 1 & -1 & 3 & 2 \\
\hline LAN-388PXCML-278 & 15 & 8 & 9 & 5 & -1 & -5 & 2 & 2 & 6 & 3 & 4 & -1 \\
\hline LAN-388PXCML-247 & 19 & 8 & 13 & 6 & 8 & 5 & 4 & 3 & 1 & -1 & -1 & -6 \\
\hline LAN-388PXCML-318 & 12 & 7 & 3 & 2 & -3 & -5 & 4 & 3 & -4 & -5 & 10 & 9 \\
\hline LAN-388PXCML-319 & 11 & 7 & 3 & 2 & -1 & -6 & 1 & -1 & -3 & -5 & 12 & 6 \\
\hline LAN-388PXCML-271 & 14 & 3 & 5 & -2 & 0 & -5 & 4 & 1 & 2 & -3 & 2 & -1 \\
\hline LB-32XCML-271 & 17 & 8 & 11 & 9 & 7 & 3 & 2 & 0 & -11 & -11 & 7 & 6 \\
\hline LB-40XCML-318 & 11 & 10 & 6 & 2 & 7 & 3 & 1 & -2 & 5 & 3 & -4 & -7 \\
\hline LAN-123XCML-318 & 21 & 11 & 5 & 0 & -4 & -5 & 1 & -1 & 9 & 7 & 5 & 2 \\
\hline LAN-388PXCML-313 & 12 & 0 & 2 & -6 & 20 & 20 & 2 & -1 & -1 & -5 & 4 & -1 \\
\hline LB-32XCML-273 & 12 & 5 & -2 & -4 & -6 & -8 & 0 & -1 & -5 & -8 & -1 & -2 \\
\hline LAN-388PXCML-321 & 12 & -2 & 3 & -5 & -2 & -4 & 2 & 0 & 1 & 1 & 3 & -4 \\
\hline
\end{tabular}

$\mathrm{RG}=$ Rendimiento de grano, $\mathrm{RMZ}=$ Rendimiento de mazorca, $\mathrm{LMZ}=$ Longitud de la mazorca, $\mathrm{DMZ}=$ Diámetro de la mazorca, $\mathrm{NGH}=$ Número de granos por hilera, $\mathrm{NHM}=$ Número de hileras por mazorca.

cruzas se observó que el RG, RMZ, LMZ y DMZ tienen significancia estadística, lo que indica que el diámetro de mazorca y el número de granos por hilera influyen en el rendimiento de mazorca y grano. Los efectos de aptitud combinatoria general para rendimiento de grano en las líneas LAN-388P, LB32 y LB-40 son altos de acuerdo con Vergara et al. (2005). Para las líneas LAN-388P y LB-32 el diámetro de mazorca y número de hileras por mazorca fueron las variables que más contribuyeron a la expresión de ACG. Al respecto Souza et al. (2009) señalan que el medio ambiente influye de manera de diferente en el comportamiento de los progenitores y por lo tanto en el valor de ACG. La ACG de las líneas hembra CML-319 (1.33) y CML-318 (1.25) tuvo valores altos, mientras que la línea CML-278 (0.88) tuvo un valor intermedio y el resto de las líneas hembras tuvieron valores bajos de acuerdo con Vergara et al. (2005). Para la línea hembra CML-319 la longitud de mazorca fue el componente que más contribuyó a la expresión de la ACG y para la línea hembra CML-318 el diámetro de mazorca, número de hileras por mazorca y longitud de mazorca fueron las variables que más contribuyeron a 
la ACG. Los valores de ACG de los progenitores machos y hembras, tuvieron mayor contribución a la expresión fenotípica, lo que coincide con De la Cruz et al. (2003).

Al comparar los valores de ACE de las cruzas, las que tuvieron los valores más altos de rendimiento no presentaron valores más altos de $A C E$, lo que se contrapone al supuesto de que valores altos de ACE se asocia con alto rendimiento (Reyes et al. 2004). Al respecto Hasan et al (2014) mencionan que esto ocurre cuando en las cruzas actúan los tipos de acción génica aditiva $x$ dominante $y$ dominante $x$ dominante. Los efectos altos de ACE de las cruzas LB-40xCML-319, LAN123xCML-278 y LAN-123xCML-318 para RG y RMZ coinciden con efectos positivos de ACG de al menos uno de los progenitores. Al respecto Ávila et al. (2009) mencionan que una cruza simple es de alto rendimiento si sus líneas progenitoras son de alta ACG o si el efecto de ACE es alto y al menos uno de sus progenitores es de alta ACG. Las cruzas LAN-388PxCML-318, LAN-388PxCML319 y LB-40xCML-318 tuvieron valores negativos de ACE para $R G$, mientras que sus progenitores tuvieron valores positivos y altos de ACG; lo que sugiere que la manifestación del alto rendimiento en las cruzas se debe a la acción génica de efectos aditivos de las líneas, como lo señalan Reyes et al. (2004). En las cuatro cruzas con valores altos de ACE para $R G$, los efectos no aditivos fueron los más importantes; con excepción de la cruza LB-40xCML-319 donde los efectos aditivos y no aditivos tuvieron importancia similar. También en las tres cruzas con valores negativos de ACE para RG, los efectos aditivos fueron importantes y positivos, contrario a los resultados obtenidos por Vergara et al. (2005). Mientras que De la Cruz et al. (2010) encontraron que la acción génica no aditiva es el componente genético más importante del rendimiento de grano. Las cruzas LB-32xCML-319 y LAN-388PxCML-278 tuvieron un comportamiento inferior a lo que se podía esperar con la ACG de sus progenitores, por el contrario, en las cruzas LAN-388PxCML-264 y LAN-123xCML-278 en las que al menos uno de sus progenitores tuvo bajo valor de ACG, tuvieron efec- tos de ACE positivos y mayores a cero; lo que sugiere que la acción génica no aditiva está involucrada en las cruzas y que el rendimiento de grano puede incrementarse mediante hibridación (De la Cruz et al. 2009). La línea LAN-388P tuvo el mayor valor de ACG como macho, e intervino en ocho de las 15 cruzas con mayor ACE. La línea CML-318 que tuvo valor positivo de ACG intervino en tres de las 15 cruzas con mayor rendimiento y efectos de ACE; por lo que estas líneas son las más indicadas para separar el resto de las líneas en dos grupos heteróticos que pudieran ser utilizados como fuente de germoplasma en programas de desarrollo de híbridos (Malacarne y San Vicente 2003).

La mayor expresión heterótica con respecto al progenitor medio y superior, la tuvieron las cruzas LB-40xCML-319 y LB-32xCML-319 cuyo origen es un grupo de líneas no emparentadas que difieren en su origen genético. La heterosis resultante de los mejores progenitores, sugiere variabilidad genética entre las poblaciones (Gutierrez et al. 2002). La diversidad genética de los tres grupos de líneas, indica qué al combinar caracteres contrastantes, se incrementa el rendimiento y sus componentes (Wong et al. 2007).

\section{CONCLUSIONES}

La mayor aptitud combinatoria general para rendimiento de grano, se encontró en las líneas macho LAN-388P, LB-32 y LB-40 y en las líneas hembras fue en CML-319, CML-318 y CML278. La mayor ACE para rendimiento de grano se tuvo en las cruzas LAN-123xCML-278, LAN 123XCML-318, LB-40xCML-319, LB-32xCML-319 y LAN-388PxCML-264. Mientras que el mayor rendimiento de grano lo tuvieron las cruzas LB40xCML-319, LB-32xCML-319, LAN-388PxCML264, LAN-123xCML-278 y LAN 123XCML-318. En relación con la heterosis, se observó que las cruzas con los valores más altos de heterosis fueron LB40xCML-319, LB-32xCML-319, LAN-388PxCML264, LAN-123xCML-278 y LAN 123XCML-318. 


\section{LITERATURA CITADA}

Antuna GO, Rincón S, Gutiérrez RE, Ruiz T NA, Bustamante G (2003) Componentes genéticos de caracteres agronómicas y de calidad fisiológica de semillas en líneas de maíz. Revista Fitotecnia Mexicana 26: 1117.

Ávila PM, Rodríguez HS, Vázquez BM, Borrego EF, Lozano RA, López BA (2009) Aptitud combinatoria y efectos recíprocos en líneas endogámicas de maíz de valles altos del centro de México. Agricultura Técnica en México 35: 285 - 293.

Bello OB, Olaoye G (2009) Combining ability for maize grain yield and other agronomic characters in a typical southern guinea savanna ecology of Nigeria. African Journal of Biotechnology 8: 2518-2522.

Comstock RE, Robinson HF (1948) The components of genetic variance in populations of biparental progenies and their use in estimating the average degree of dominance. Biometrics 4: 254-266.

De la Cruz LE, Gutiérrez RE, Palomo GA, Rodríguez HSA (2003) Aptitud combinatoria y heterosis de líneas de maíz en la Comarca Lagunera. Revista Fitotecnia Mexicana 26: 279-284.

De la Cruz LE, Córdova OH, Estrada BMA, Mendoza PJD, Gómez VA, Brito MNP (2009) Rendimiento de grano de genotipos de maíz sembrados bajo tres densidades de población. Universidad y Ciencia 25: 93-98.

De la Cruz LE, Castañón NG, Brito MNP, Gómez VA, Robledo TV, Lozano RAJ (2010) Heterosis y aptitud combinatoria de poblaciones de maíz tropical. Revista Internacional de Botánica Experimental 79: 11-17.

De León CH, Rincon-Sánchez F, Reyes-Valdez HM, Sámano-Garduño D, Martínez-Zambrano G, CavazosCadena R, et al. (2005) Potencial de rendimiento y estabilidad de combinaciones germoplásmicas formadas entre grupos de maíz. Revista Fitotecnia Mexicana 28: 135-143.

Echarte L, Andrade HF, Vega, M Tollenaar CR (2004) Kernel number determination in argentinian maize hybrids released between 1965 and 1993. Crop Science 44: 1654-1661.

Guillen-De la Cruz P, De la Cruz-Lázaro E, Castañón-Nájera G, Osorio-Osorio R, Brito-Manazano NP, Lozanodel Río A, et al. (2009) Aptitud combinatoria general y específica de germoplasma tropical de maíz. Tropical and Suptropical Agroecosystems 10: 101-107

Gutiérrez RE, Palomo GA, Espinoza BA, De la Cruz LE (2002) Aptitud combinatoria y heterosis para rendimiento de líneas de maíz en la camara lagunera, México. Revista Fitotecnia Mexicana 25: 271277.

Hasan MJ, Kulsum MU, Rahman MM (2014) Combining ability of different yield related characters in rice. SAARC Journal of Agriculture 12: 143-153.

Manjarrez SM, Palemón AF, Orlando GMN, Espinosa CA, Rodríguez HSA, Damián NA, et al. (2014) Aptitud combinatoria general y específica de maíces normales y de alta calidad de proteína. Revista Mexicana de Ciencias Agrícolas 5: 1261-1273.

Malacarne MF, San Vicente GFM (2003) Patrones heteróticos de líneas tropicales blancas de maíz. Agronomía Tropical 53: 32-40.

Rehman IN, Shah NH, Arif M, Siddiq M Mian IA (2011) Correlations among grain yield and yield attributes in maize hybrids in various nitrogen levels. Sarhad Journal of Agricultural 27: 531-538. 
Reyes LD, Molina GJD, Oropeza RMA, Moreno PEC (2004) Cruzas dialélicas entre líneas autofecundadas de maíz derivadas de la raza tuxpeño. Revista Fitotecnia Mexicana 27: 49-56.

Sprague GF, Tatum LA (1942) General versus specific combining ability in single crosses of corn. Journal American of Society of Agronomy 34: 923-932.

SAS (1988) Statistical Analysis System. SAS/STAT User's Guide: release 6.11. SAS Institute Inc. Cary, NC. USA.

Shawn K (2012) Heterosis: Many genes, many mechanisms? End the search for an undiscovered unifying theory. International Scholarly Research Network, International Scholarly Research Notices Botany. Article ID 682824, 12p.

Souza LV de, Miranda GV, Cardoso GJC, Moreira GLJ, Santos IC dos (2009) Combining ability of maize grain yield under different levels of environmental stress. Pesquisa Agropecuária Brasileira 44: 1297-1303.

Sylvain G (2010) Surprising fitness consequences of GC-biased gene conversion. II. Heterosis. Genetics 187: 217-227.

Vergara AN, Rodríguez HSA, Córdoba OH (2005) Aptitud combinatoria general y específica de líneas de maíz (Zea Mays) tropical y subtropical. Agronomía Mesoamericana 16: 137-143.

Wong RR, Gutiérrez RE, Palomo GA, Rodríguez HS, Córdoba OH, Espinoza BA, et al. (2007) Aptitud combinatoria de componentes del rendimiento en líneas de maíz para grano en la Comarca Lagunera, México. Revista Fitotecnia Mexicana 30: 181-189.

Zhang X, Liangjie Lv, Chao Lv, Baojian B, Rugen X (2015) Combining ability of different agronomic traits and yield components in hybrid barley. PLoS ONE 10(6): e0126828. 